\title{
Afferent Connections of the Valvula Cerebelli in Two Teleosts, the Common Goldfish and the Green Sunfish
}

\author{
MARIO F. WULLIMANN AND R. GLENN NORTHCUTT \\ Neurobiology Unit, Scripps Institution of Oceanography and Department of Neurosciences \\ A-001, University of California San Diego, La Jolla, CA 92093
}

\begin{abstract}
The afferent connections of the valvula cerebelli were examined in one cypriniform teleost (Carassius auratus) and one perciform teleost (Lepomis cyanellus) with the use of horseradish peroxidase as a retrograde tracer. Both species have ipsilateral input to the valvula from the central pretectal and dorsal accessory optic nuclei, the dorsal and ventral tegmental nuclei, the lateral nucleus of the valvula, the perilemniscal nucleus, and nucleus isthmi and contralateral input from the inferior olivary nucleus. In addition, Carassius has ipsilateral valvulopetal projections from the eminentia granularis, the praeeminential nucleus, and the isthmic primary sensory trigeminal nucleus, whereas Lepomis has bilateral (stronger ipsilaterally) valvulopetal projections from the nucleus of the locus coeruleus and the rostral corpus cerebelli.

The topographical order of the cerebellopetal projections of the lateral nucleus of the valvula and inferior olive is also described, as are differential inputs to various subdivisions of the cerebellum in the two species.

Information on valvulopetal projections in teleosts has thus far been limited to electroreceptive mormyrids. The present study shows that many valvular inputs related to electroreception in mormyrids have no homologue in $\mathrm{Car}$ assius and Lepomis.

Finally, the present study indicates that the rostral part of the corpus cerebelli, but not the valvula cerebelli, in teleosts is the homologue of the anterior lobe of the corpus cerebelli in cartilaginous fishes. Thus, the valvula cerebelli is a shared derived feature (synapomorphy) of all ray-finned fishes.
\end{abstract}

Key words: electroreception, evolution, fish, sensory systems, topography, valvulopetal

The valvula cerebelli is a subdivision of the cerebellum that forms a rostral protrusion extending below the optic tectum into the mesencephalic ventricle (Figs. 1, 2). It is found exclusively in ray-finned fishes (Actinopterygii) (Larsell, '67; Nieuwenhuys, '67, '82), the largest group of which are the teleosts. The most extensive development of the valvula cerebelli occurs in African mormyrid teleosts (Nieuwenhuys and Nicholson, '69a), where anatomical (Finger et al., '81) and electrophysiological (Russell and Bell, '78) evidence indicates that major parts of the valvula are devoted to the processing of electrosensory stimuli. Comparable data on the valvula cerebelli in ray-finned fishes other than mormyrids do not exist.

Electroreception was initially present in actinopterygians (cladistians and chondrosteans), but it was subsequently lost in neopterygians (Bullock et al., '83). Mormyrids are one of the few groups of teleosts that have reevolved the electrosensory modality (Bullock et al., '83). Involvement of the valvula cerebelli in the processing of electrosensory information is thus a uniquely derived feature (autapomorphy) for mormyrids and clearly represents a case of exaptation, not adaptation (see Gould and Vrba, '82, for discussion of the concept of exaptation).

It is therefore important to determine the anatomic connections of the valvula cerebelli in teleosts that are not electroreceptive, as these connections are likely to represent the

Accepted June 8, 1989.

Address reprint requests to Dr. Mario F. Wullimann, Georg-August-Universität, Zentrum Anatomie, Kreuzbergring 36, D-3400 Göttingen, West Germany. 
primitive condition for teleosts. We examined anatomical inputs to the valvula cerebelli in two nonelectroreceptive teleosts: the common goldfish, Carassius auratus (Ostariophysinae) and the green sunfish, Lepomis cyanellus (Percomorpha), in which considerable details are known regarding extravalvular neuronal circuits (Northcutt and Wullimann, '88).

\section{Abbreviations}

\begin{tabular}{|c|c|}
\hline A & nucleus anterior thalami \\
\hline $\mathrm{AC}$ & anterior cerebellar tract \\
\hline $\mathrm{CCe}$ & corpus cerebelli \\
\hline $\mathrm{CH}$ & commissura horizontalis \\
\hline $\mathrm{CM}$ & corpus mammillare \\
\hline CPN & nucleus pretectalis centralis \\
\hline DT & nucleus tegmentalis dorsalis \\
\hline $\mathrm{EG}$ & eminentia granularis \\
\hline EW & perinuclear neurons of the nucleus of Edinger-Westphal \\
\hline FLL & fasciculus longitudinalis lateralis \\
\hline FLM & fascululus longitudinalis medialis \\
\hline FLo & facial lobe \\
\hline G & granular layer of cerebellum \\
\hline Hy & hypophysis \\
\hline IO & inferior olivary nucleus \\
\hline IR & inferior raphe \\
\hline IRF & inferior reticular formation \\
\hline $\mathrm{LC}$ & nucleus of the locus coeruleus \\
\hline LCe & lobus caudalis cerebelli \\
\hline LI & lobus inferior hypothalami \\
\hline $\bar{M}$ & molecular layer of cerebellum \\
\hline NAOD & nucleus accessorius opticus dorsalis \\
\hline $\mathrm{NC}$ & nucleus corticalis \\
\hline NCLI & nucleus centralis of the inferior lobe \\
\hline NDLIC & nucleus diffusus of the inferior lobe, pars caudalis \\
\hline NDLIl & nucleus diffusus of the inferior lobe, pars lateralis \\
\hline NDLIm & nucleus diffusus of the inferior lobe, pars medialis \\
\hline $\mathrm{Ne}$ & nervus vagus \\
\hline NGp & nucleus glomerulosus pars posterior \\
\hline NGS & nucleus gustatorius secundarius \\
\hline NI & nucleus isthmi \\
\hline NIn & nucleus interpeduncularis \\
\hline NLV & nucleus lateralis valvulae \\
\hline NMT & nucleus motorius nervi trigemini \\
\hline $\mathrm{NOc}$ & nucleus nervi oculomotorius \\
\hline NPLI & $\begin{array}{l}\text { nucleus periventricularis recessus lateralis of the inferior } \\
\text { lobe }\end{array}$ \\
\hline NTP & nucleus tuberis posterior \\
\hline ON & nervus opticus \\
\hline $\mathrm{PE}$ & nucleus prae-eminentialis \\
\hline $\mathrm{PGl}$ & nucleus preglomerulosus pars lateralis \\
\hline PL & nucleus perilemniscularis \\
\hline $\mathrm{PPp}$ & nucleus preoptieus parvocellularis posterioris \\
\hline $\mathrm{PSi}$ & nucleus pretectalis superficialis pars intermedius \\
\hline PSm & nucleus pretectalis superficialis pars magnocellularis \\
\hline SCh & nucleus suprachiasmaticus \\
\hline SG & nucleus subglomerulosus \\
\hline STN & isthmic primary sensory trigeminal nucleus \\
\hline SV & saccus vasculosus \\
\hline TA & nucleus tuberis anterior \\
\hline Tel & telencephalon \\
\hline $\mathrm{TeO}$ & tectum opticum \\
\hline TGS & tractus gustatorius secundarius \\
\hline TL & torus longitudinalis \\
\hline TLa & nucleus of the torus lateralis \\
\hline TMCa & tractus mesencephalo-cerebellaris anterior \\
\hline TO & tractus opticus \\
\hline TS & torus semicircularis \\
\hline TTB & tractus tecto-bulbaris \\
\hline $\mathrm{Va}$ & valvula cerebelli \\
\hline Val & valvula cerebelli, lateral division \\
\hline Vam & valvula cerebelli, medial division \\
\hline VL & nucleus ventrolateralis thalami \\
\hline VLo & vagal lobe \\
\hline VM & nucleus ventromedialis thalami \\
\hline VT & nucleus tegmentalis ventralis \\
\hline
\end{tabular}

A second question is also addressed in this study. In cartilaginous fishes, the out-group of actinopterygian and sarcopterygian fishes, the corpus cerebelli comprises an anterior lobe and a posterior lobe (Northcutt, '77, '78), which receive different inputs (Northcutt and Brunken, '84; Fiebig, '88). It can be hypothesized that the anterior cerebellar lobe of cartilaginous fishes represents the homologue of the valvula cerebelli in ray-finned fishes, as each is the most rostral subdivision of the cerebellum. In this case, only the unusual position of the valvula below the optic tectum would be a uniquely derived feature (autapomorphy) for actinopterygians; the structure itself would have a homologue in cartilaginous fishes. If the differential connections of the valvula and corpus cerebelli in teleosts are similar to the differential connections of the anterior lobe and posterior lobe of the corpus cerebelli in cartilaginous fishes, this hypothesis of homology would be supported. If differential connections of the cerebellar subdivisions are not similar in teleosts and cartilaginous fishes, this would be evidence that the valvula cerebelli is an evolutionary innovation of the cerebellum shared by all actinopterygians (Nieuwenhuys, '67) and constituting a synapomorphy for this group.

The purpose of this study was therefore twofold: 1) to establish the connections of the valvula cerebelli in two nonelectroreceptive teleost species to determine if and how these connections differ from those in electroreceptive mormyrids; and 2) to determine if the differential connections of the valvula and corpus cerebelli in ray-finned fishes resemble the differential connections of the anterior lobe and posterior lobe of the corpus cerebelli in cartilaginous fishes.

Unless otherwise noted, the neuroanatomical nomenclature is that of Braford and Northcutt ('83), Northcutt and Wullimann ('88), and Wullimann and Northcutt ('88a). The taxonomic terminology is according to Lauder and Liem ('83). The afferent connections of the valvula cerebelli in Carassius auratus were briefly described previously (Wullimann and Northcutt, ' $88 b)$.

\section{MATERIAIS AND METHODS Animals}

Common goldfish(Carassius auratus, 0.06-0.15 m standard length), and green sunfish (Lepomis cyanellus, 0.05$0.1 \mathrm{~m}$ standard length) were obtained from local dealers, maintained at temperatures of $22-24^{\circ} \mathrm{C}$ in filtered group tanks, and fed twice a week.

\section{Experimental procedures}

Surgical procedures were identical to those described previously (Wullimann and Northcutt, '88a) except for the techniques used to apply horseradish peroxidase (HRP, Sigma VI). In this study, HRP was pin-injected (size 000) into the valvula cerebelli after the intertectal commissure was cut, and a portion of the optic tectum was removed unilaterally to expose the valvula (Carassius, 16 cases; Lepomis, nine cases). In additional animals (Carassius, 17 cases; Lepomis, ten cases), concentrated HRP solution was pressure-injected through a pipette into the valvula cerebelli with the aid of a stereotaxic device.

After survival times of 1 to 7 days for Carassius, or 2 to 6 days for Lepomis, the animals were reanesthetized in tricaine methanesulfonate (MS 222, Sigma) and transcardially perfused with either $0.04 \mathrm{M}$ phosphate buffer ( $\mathrm{pH} 7.4$ ) or 1\% Heparin (Elkins-Sinn) in teleost Ringer's solution followed by $4 \%$ glutaraldehyde in phosphate buffer. Histologi- 


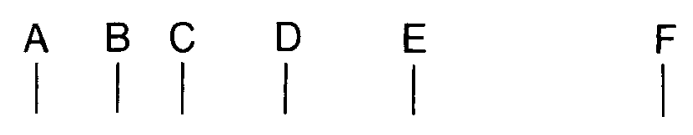

\section{A}

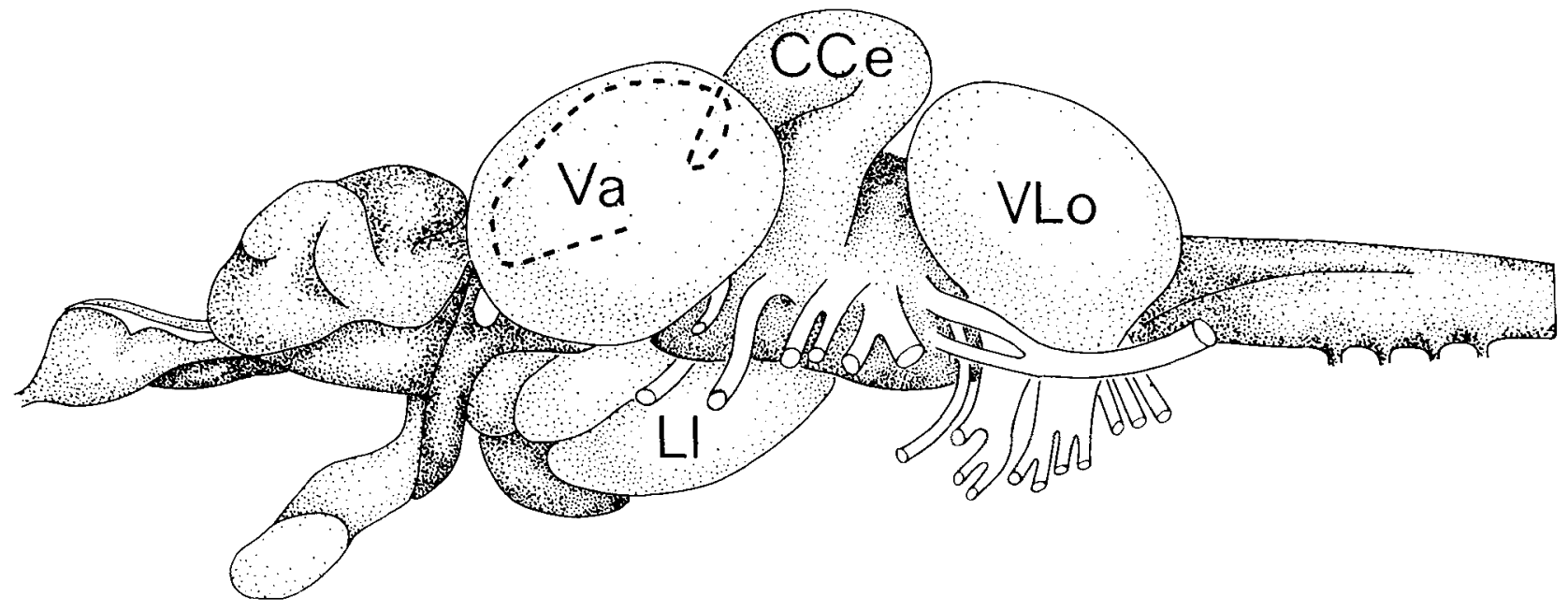

$\stackrel{i}{i} \stackrel{i}{C} \stackrel{C}{i}$

B

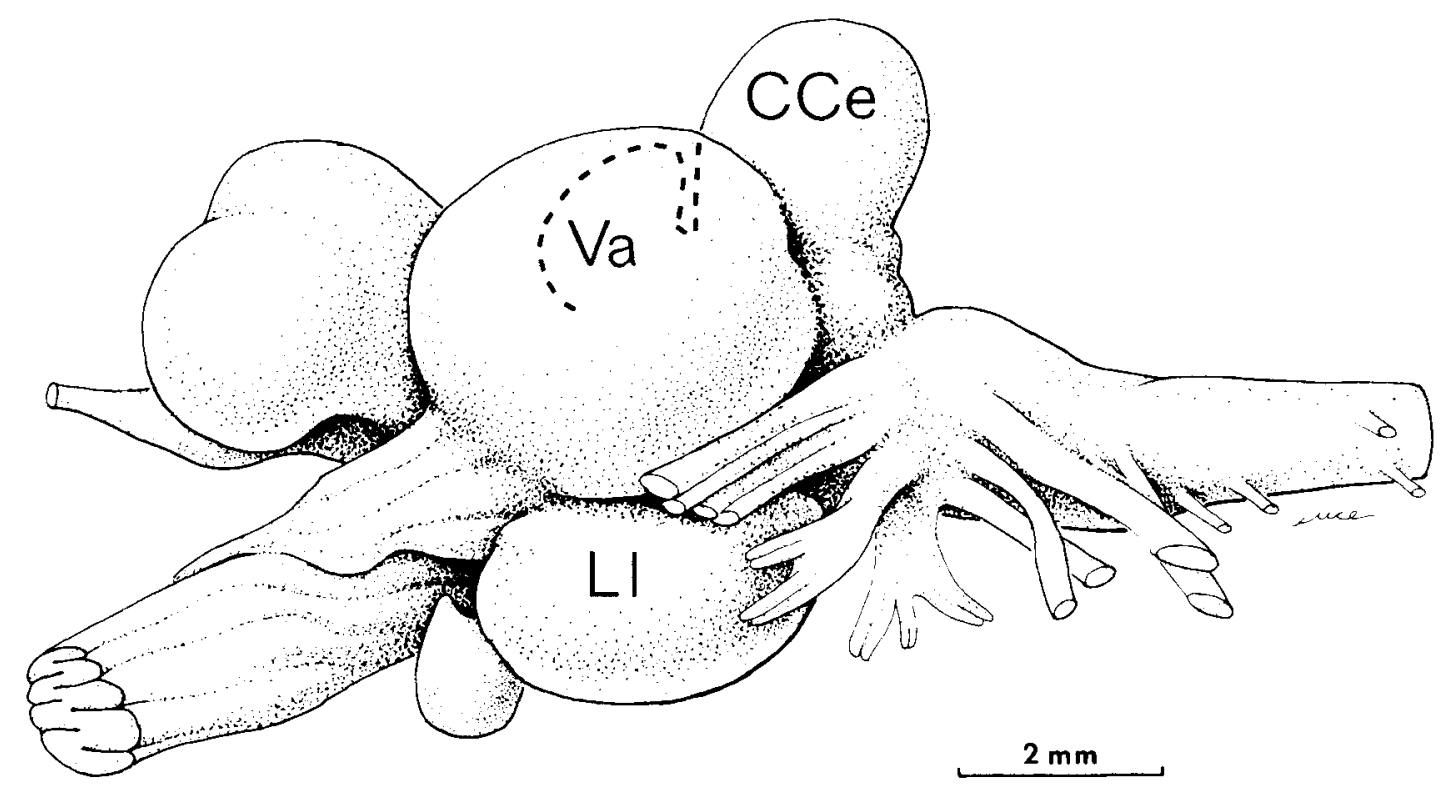

Fig. 1. Brains of Carassius auratus (A) and Lepomis cyanellus (B) in lateral view. The valvula cerebelli is outlined with dashes. Levels of charted afferent connections of the valvula cerebelli (see Figs. 3,4) are indicated. 

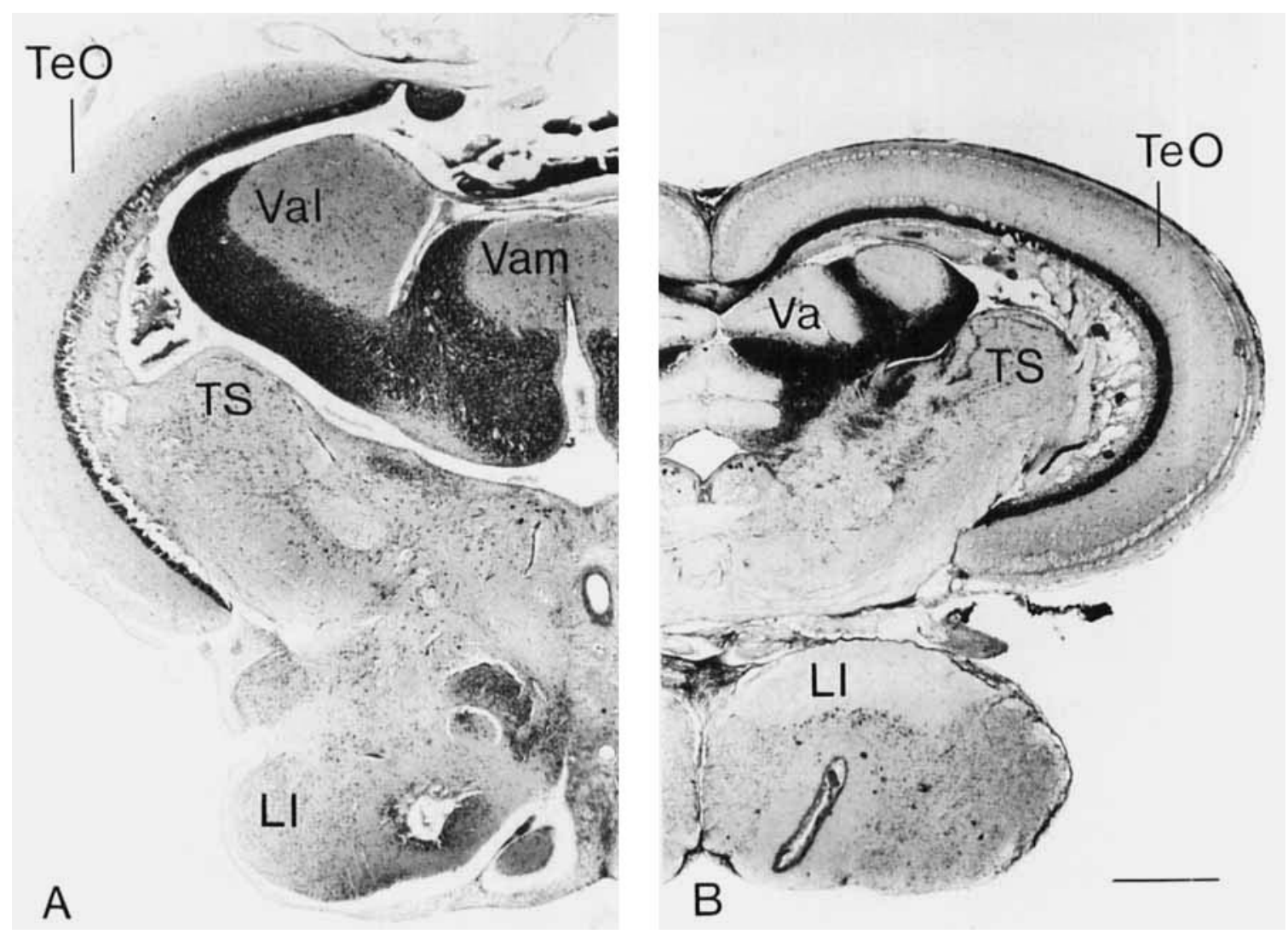

Fig. 2. Valvula cerebelli of Carassius (A) and Lepomis (B) in Bodian-stained cross sections. Note this structure's larger size in Carassius and more complex folding in Lepomis. Dorsal is to the top; medial is to the right in $A$ and to the left in $B$. Bar scale $=0.5 \mathrm{~mm}$.

cal procedures were identical to those described previously (Wullimann and Northcutt, '88a), but in the present study only the Hanker-Yates protocol (Hanker et al., '77) was used to visualize the HRP reaction. Both Hanker-Yates and tetramethyl benzidine (TMB; Mesulam, '78) protocols were used to visualize the connections of the corpus cerebelli in the same two teleost species (Wullimann and Northcutt, '88a); TMB revealed no additional labeled neurons, and the Hanker-Yates protocol yielded far more cytological detail. We therefore chose to use only the Hanker-Yates protocol to investigate the valvular afferent connections reported herein.

\section{Normal anatomy}

Bodian-stained transversely cut brains from both Carassius and Lepomis were available for study. Details of their preparation were described previously (Wullimann and Northcutt, '88a).

\section{RESULTS}

A short description of the normal morphology of the cerebellum in Carassius and Lepomis will be followed by a detailed description of the afferent connections of the valvula cerebelli in the two species, a section on the topography of some cerebellopetal connections, and finally a description of the differential anatomical inputs to the cerebellum.

\section{Normal morphology}

The three major subdivisions of the cerebellum-corpus cerebelli, valvula cerebelli, and vestibulolateral lobe-have been described previously in these species (Wullimann and Northcutt, '88a). The extent of the valvula relative to that of the corpus cerebelli is indicated in Figure 1. Although the valvula is relatively larger in Carassius than in Lepomis, it is organized more simply in the former, where it is merely separated into a medial part and a lateral part by a longitudinal fissure (Figs. 2A, 3B,C). In Lepomis, the valvula is complexly folded overall and not apparently divided into medial and lateral subdivisions (Figs. 2B, 4B,C).

\section{Afferent connections of the valvula cerebelli}

After injections into the medial part of the valvula cerebelli of Carassius, neurons in the following cell groups were found to be retrogradely filled ipsilaterally: the central pretectal nucleus and dorsal accessory optic nucleus at diencephalic levels (Fig. 3A); the dorsal and ventral tegmental nuclei and perilemniscal nucleus in the mesencephalon (Figs. 3B,C); and the lateral nucleus of the valvula, nucleus 

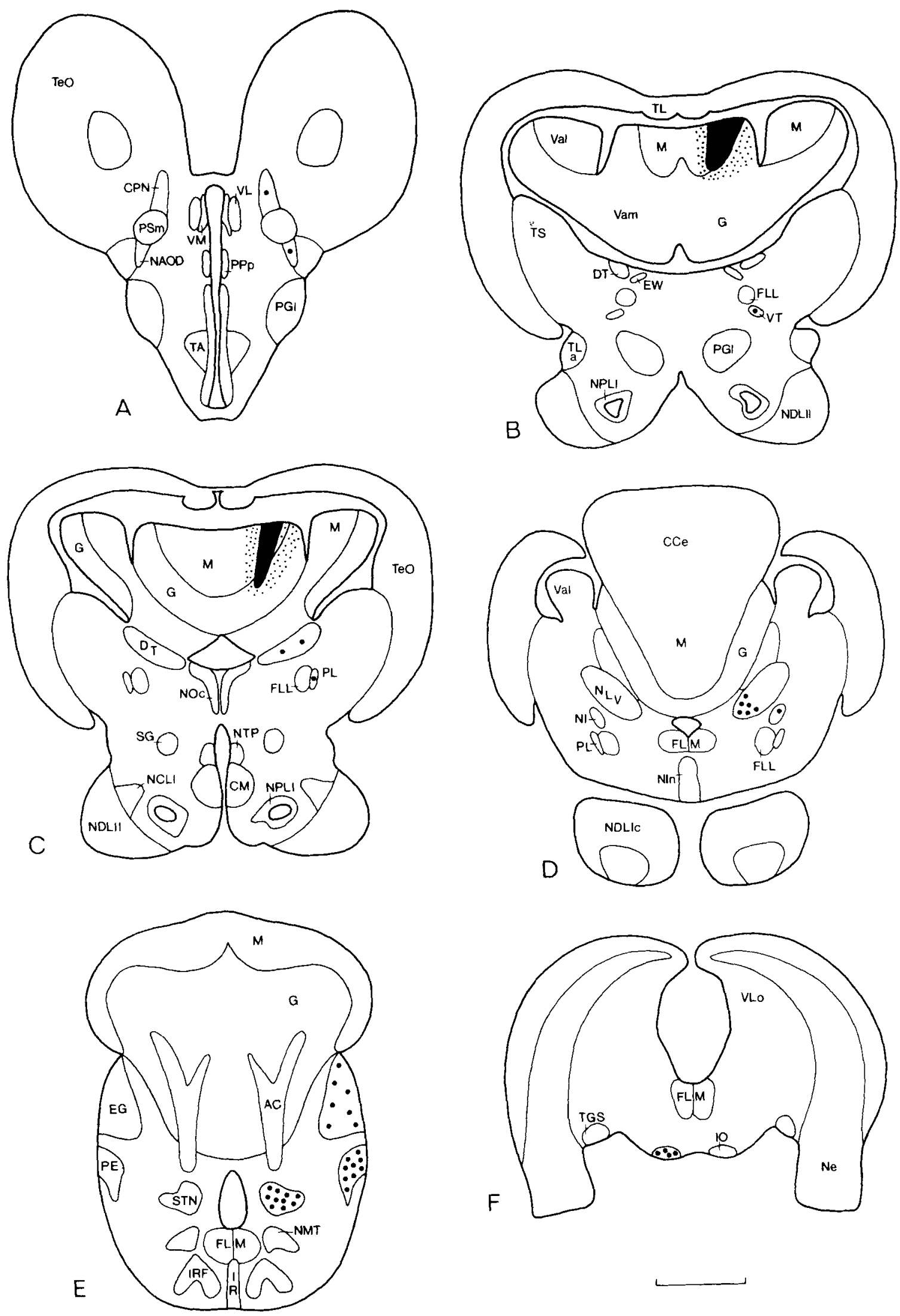

Fig. 3. Chartings of afferent connections of the valvula cerebelli in Carassius. Black dots represent retrogradely labeled cells. Black area and stippling indicate the injection site and its surrounding area. Bar scale = $1 \mathrm{~mm}$. 

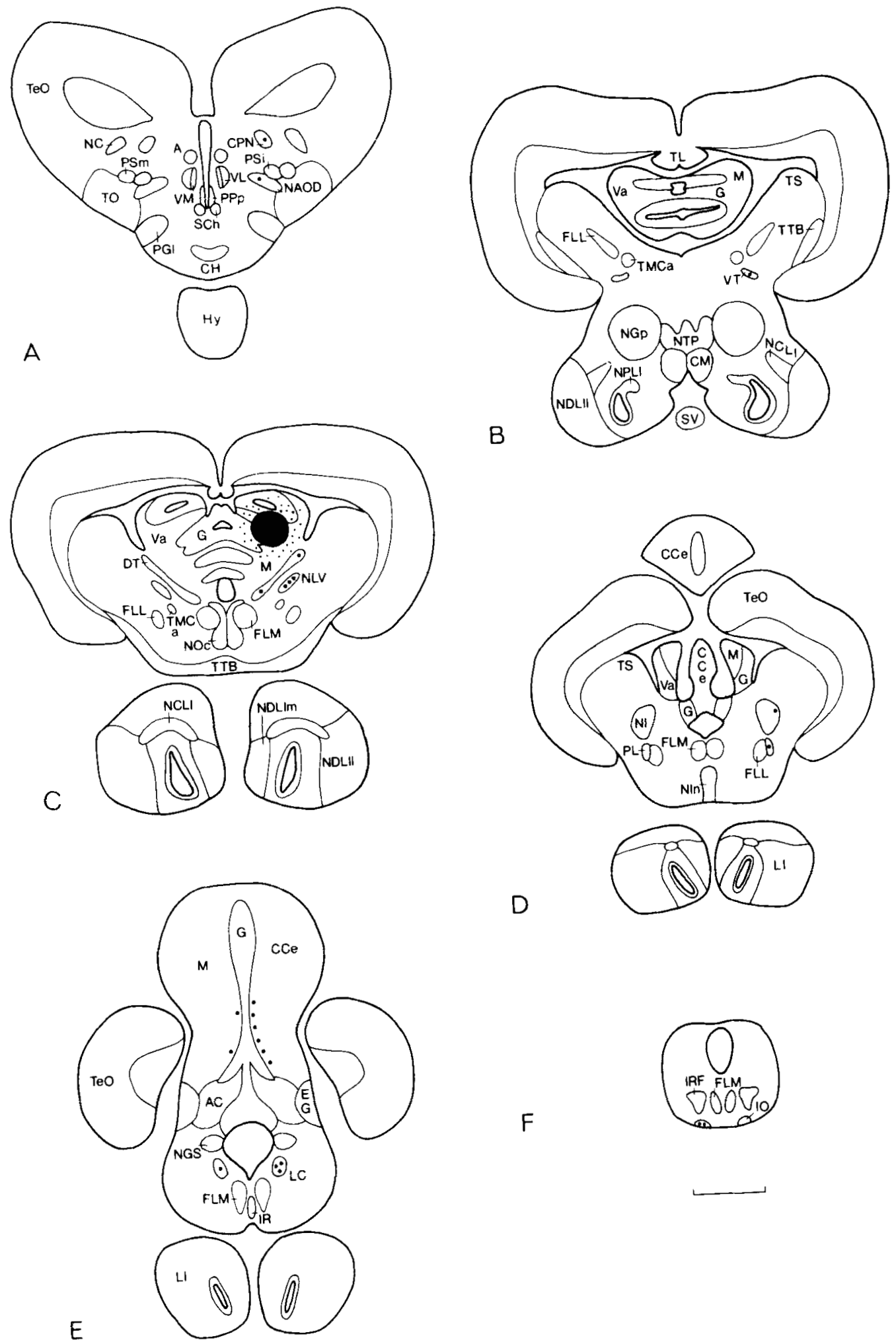

Fig. 4. Chartings of afferent connections of the valvula cerebelli in Lepomis. Black dots represent retrogradely labeled cells. Black area and stippling indicate the injection site and its surrounding area. Bar scale = $1 \mathrm{~mm}$. 
isthmi, eminentia granularis, prae-eminential nucleus, and isthmic primary sensory trigeminal nucleus at metencephalic and myelencephalic levels (Figs. 3D,E, 5A-C). The contralateral inferior olivary nucleus was labeled as well (Figs. 3F, 5E).

Injections into the lateral part of the valvula cerebelli of Carassius resulted in retrograde labeling of the same cell groups, except that no retrogradely filled cells were found in the eminentia granularis, prae-eminential nucleus and isthmic primary sensory trigeminal nucleus.

In several nuclei, fewer labeled neurons resulted from valvular than corpal injections: the central pretectal nucleus, dorsal accessory optic nucleus, dorsal and ventral tegmental nuclei, the perilemniscal nucleus, and nucleus isthmi (Wullimann and Northcutt, '88a). Retrogradely filled cells in the lateral nucleus of the valvula and the inferior olive, however, were equally numerous after injections of the valvula (Figs. $5 \mathrm{C}, \mathrm{E})$.

Injections of the valvula in Lepomis yielded a similar pattern of afferent connections (Figs. 4,6). Fewer neurons were retrogradely filled in the ipsilateral central pretectal nucleus, dorsal accessory optic nucleus, ventral tegmental nucleus, perilemniscal nucleus, and nucleus isthmi; more neurons were labeled in the ipsilateral dorsal tegmental nucleus and lateral nucleus of the valvula and the contralateral inferior olive (Fig. 4). In contrast to Carassius, retrogradely labeled neurons were also observed in the ipsilateral and (more weakly) contralateral nucleus of the locus coeruleus (Figs. 4E; 5D) and in large cells located in the Purkinje cell layer of the rostral corpus cerebelli (Fig. 4E). Although labeled fibers within the ipsilateral anterior cerebellar tract were also seen to enter the corpus cerebelli after injections of the valvula in Carassius, no retrogradely filled cells were found in the corpus cerebelli.

Also, in contrast to Carassius, labeled neurons were not seen in the eminentia granularis, the prae-eminential nucleus, and the isthmic primary sensory trigeminal nucleus in Lepomis, although both eminentia granularis and isthmic primary sensory trigeminal nucleus can be identified in normal histology in both species, and the isthmic primary sensory trigeminal nucleus can be demonstrated experimentally, as it receives primary trigeminal projections in both Carassius (Puzdrowski, '88a) and Lepomis (R.L. Puzdrowski and M.F. Wullimann, unpublished observations). However, a distinct prae-eminential nucleus, as observed in Carassius, cannot be visualized morphologically or experimentally in Lepomis.

\section{Topography}

In Carassius, at least two nuclei-the inferior olivary nucleus and the lateral nucleus of the valvula-project topographically to the valvula and corpus cerebelli (Fig. 7). The inferior olive projects upon the contralateral cerebellum in rostrocaudally reversed topographical order: the caudal part of the inferior olive projects to the rostrally located valvula cerebelli; the rostral part of the inferior olive projects to the caudally located corpus cerebelli (Fig. 7). The same topographical olivocerebellar projection exists in Lepomis. The topographical nature of the olivocerebellar connection in teleosts was reported previously (Finger, '83).

The lateral nucleus of the valvula projects topographically to the ipsilateral valvula cerebelli in Carassius. The medial and caudolateral parts of the lateral nucleus of the valvula project to the medial part of the valvula; the lateral and caudomedial parts of the lateral nucleus of the valvula project to the lateral part of the valvula cerebelli (Figs. 5C, 7). The area of the lateral nucleus of the valvula that projects to the corpus cerebelli largely overlaps that projecting to the valvula cerebelli (Fig. 7).

Because the valvula cerebelli (Figs. 2A,B) in Lepomis differs morphologically from that in Carassius (Wullimann and Northcutt, '88a), injections restricted to the lateral valvula could not be made in Lepomis, and it therefore could not be determined if Lepomis possesses comparable topographical organization of afferent connections from the lateral nucleus of the valvula to the valvula cerebelli. As in Carassius, however, the areas of the lateral nucleus of the valvula that project to the valvula cerebelli in Lepomis overlap with those projecting to the corpus cerebelli.

\section{Differential anatomical inputs to the cerebellum}

In addition to the topographical order of some of the cerebellopetal projections, there is a second category of spatially ordered afferent projections to the valvula and corpus cerebelli: not all cerebellopetal nuclei project uniformly to all parts of the cerebellum. This pattern of differential input to the cerebellum is summarized in Figure 8 . In both Carassius and Lepomis, nuclei that project to the corpus cerebelli comprise an anterior group-located in the diencephalon, mesencephalon, and rostral metencephalon-and a posterior group that is situated in the rhombencephalon (Wullimann and Northcutt, '88a). In both species, some nuclei of the anterior group also project to the valvula cerebelli: central pretectal nucleus, dorsal accessory optic nucleus, dorsal and ventral tegmental nuclei, lateral nucleus of the valvula, nucleus isthmi, and perilemniscal nucleus in both Carassius (Figs. 6A, 8) and Lepomis (Fig. 6B) plus the nucleus of the locus coeruleus in Lepomis. However, judging from the number of retrogradely filled neurons, only the lateral nucleus of the valvula in Carassius and the dorsal tegmental nucleus and lateral nucleus of the valvula in Lepomis project as heavily to the valvula as to the corpus cerebelli. Of the posterior group of corpopetal nuclei, only the inferior olive projects to both the valvula and the corpus cerebelli in the two species. An additional group of isthmic nuclei (praeeminential nucleus, isthmic primary sensory trigeminal nucleus, eminentia granularis) project to the valvula, but not to the corpus cerebelli, in Carassius only (Figs. 6A, 8).

Due to the caudally directed bending of the corpus cerebelli in both Carassius and Lepomis (Fig. 1), the dorsal surface of the corpus actually corresponds topologically to the rostral surface, the caudal tip of the corpus corresponds topologically to the dorsal surface, and the ventral part of the corpus corresponds topologically to the caudal part. The conventional axes used to indicate morphological position therefore do not correspond to topologically correct positions in the corpus. In Figure 8 both conventional and distorted axes are indicated, as their relationship is important in comparing the differential inputs from the anterior and posterior groups of corpopetal nuclei. As all injections into the corpus were made from the dorsal aspect, they necessarily included the topologically rostral part of the corpus. Only injections that included the ventral (topologically caudal) parts of the cerebellar corpus resulted in retrograde labeling of posterior cell groups (Wullimann and Northcutt, '88a), with one exception: the inferior olive was also labeled after restricted injections into the dorsal (topologically ros- 

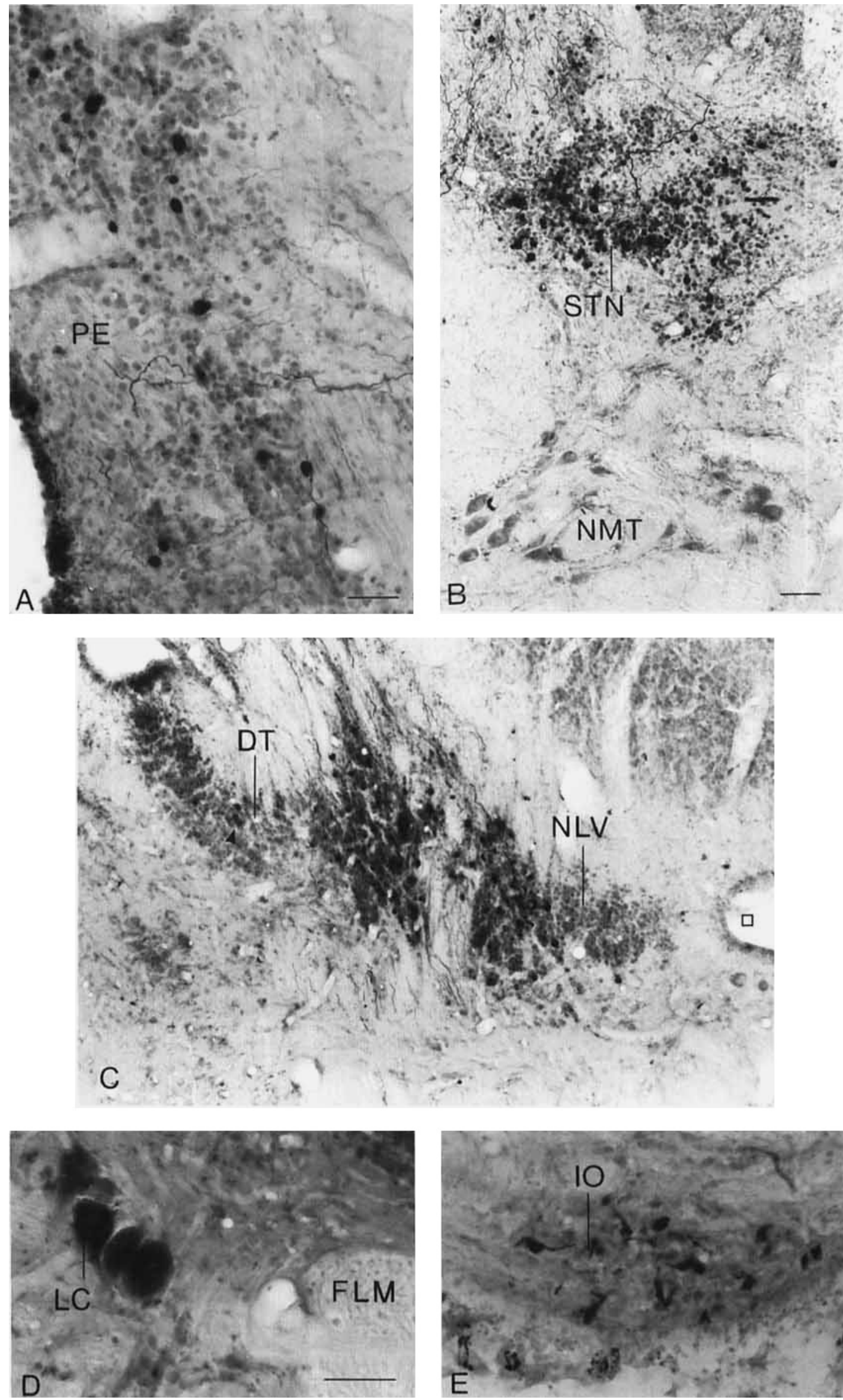

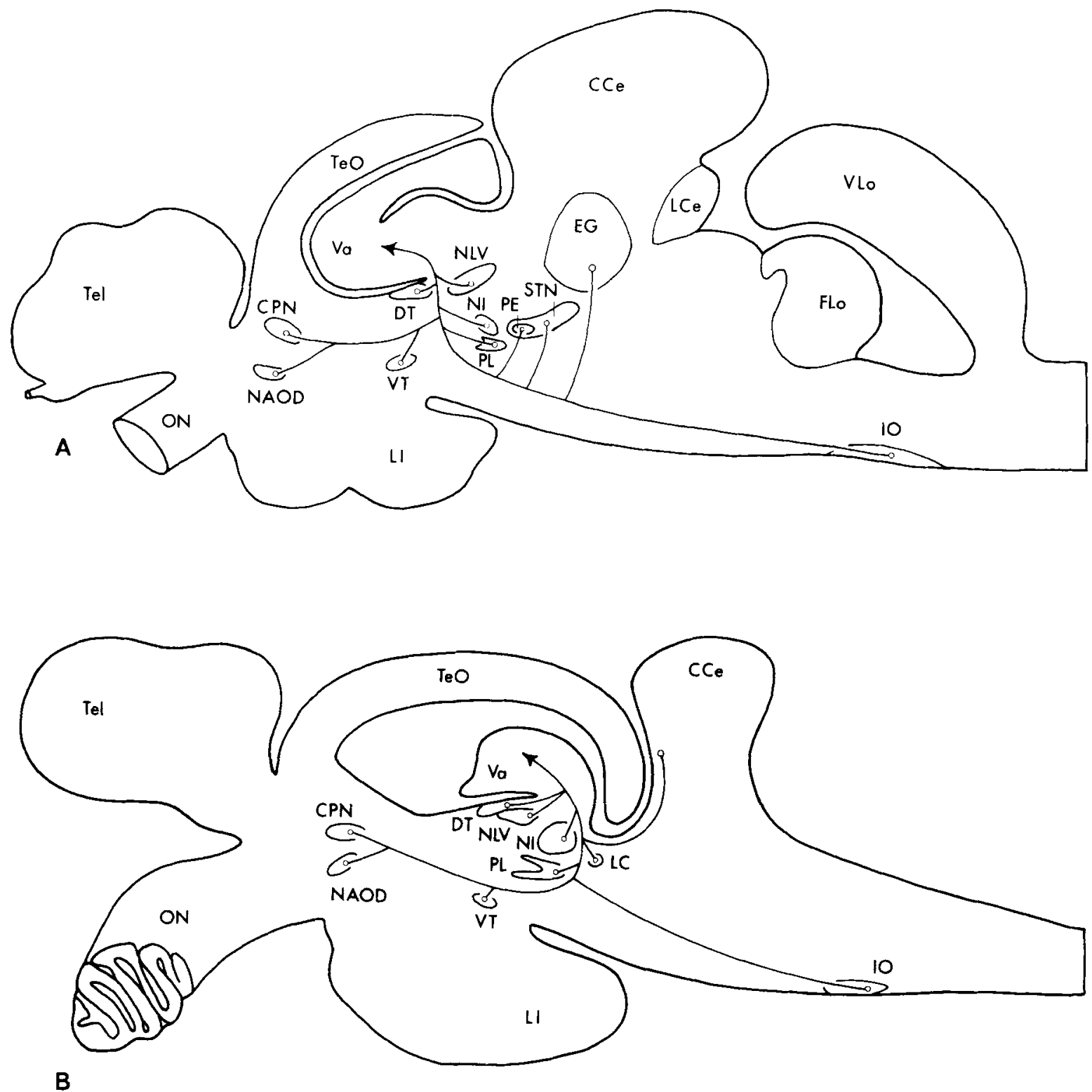

Fig. 6. Schematic diagram summarizing afferent connections of the valvula cerebelli in Carassius (A) and Lepomis (B). Note that the isthmic group of valvulopetal nuclei is present only in Carassius.

Fig. 5. Valvulopetal nuclei in Carassius (A-C,E) and Lepomis (D) visualized after HRP injections into the valvula cerebelli. A,B: Two main isthmic valvulopetal nuclei, the nucleus prae-eminentialis, and the isthmic primary sensory trigeminal nucleus. C: After a lateral injection into the valvula, only the lateral part of the lateral nucleus of the valvula is labeled. Some labeled neurons (arrowhead) in the dorsal tegmental nucleus can also be seen. D: Large neurons of the nucleus of the locus coeruleus are labeled only in Lepomis. E: Note that neurons throughout the dorsoventral extent of the inferior olive are labeled. All photomicrographs are of cross-sections. Lateral is to the left (except in E, where lateral is to the right) and dorsal is to the top. The ventricle is indicated with a square. Bar scale $=0.05 \mathrm{~mm}$ in $\mathrm{A}$ and $\mathrm{D}$ (same magnification in $\mathrm{E}$ ) and $0.1 \mathrm{~mm}$ in $\mathrm{B}$ (same magnification in $\mathrm{C}$ ). tral) part of the corpus. It is thus clear that the posterior group of corpopetal nuclei (except the inferior olive) project exclusively to the ventral (topologically caudal) part of the corpus cerebelli (Fig. 8). Because none of our injections were restricted to the ventral (topologically caudal) part of the corpus cerebelli, it is not clear if the anterior group of corpopetal nuclei and the inferior olive also project exclusively to the dorsal (topologically rostral) part of the corpus.

An additional pattern of differential anatomical inputs to the valvula cerebelli can be observed in Carassius: The medial part of the valvula receives input from all valvulopetal nuclei observed in this study, but the lateral part of the 


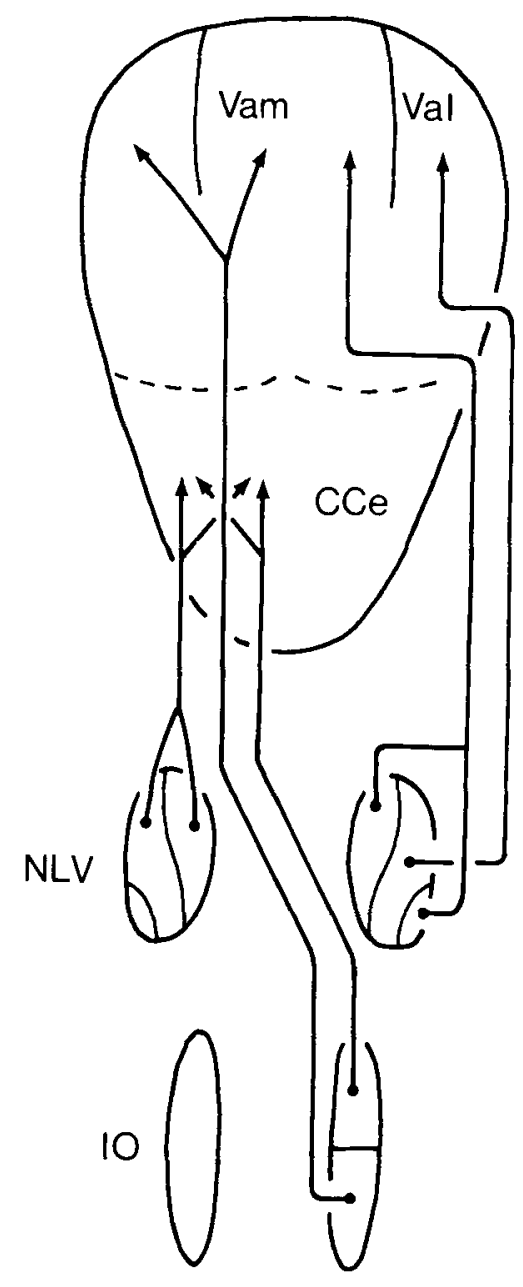

Fig. 7. Schematic diagram summarizing the topographic projections of the lateral nucleus of the valvula and the inferior olive to the cerebellum in Carassius. Dashes indicate the outline of the optic tectum. Rostral is to the top of the figure, caudal to the bottom.

valvula does not receive input from the isthmic group (isthmic primary sensory trigeminal nucleus, prae-eminential nucleus, eminentia granularis). No differential inputs to specific valvular subdivisions were observed in Lepomis.

\section{DISCUSSION}

The afferent connections of the valvula cerebelli in Carassius and Lepomis will be discussed first, followed by consideration of the first question posed in the Introduction: Are the afferent connections of the valvula cerebelli different in electrosensory and nonelectrosensory teleosts? The differential anatomical inputs to the cerebellum will be discussed next, followed by consideration of the second question posed in the Introduction: Does the valvula cerebelli have a homologue in cartilaginous fishes?

\section{Afferent connections of the valvula cerebelli}

Injection of HRP into the valvula cerebelli requires that the optic tectum be ablated unilaterally (pin injections) or penetrated (pipette injections). 'Therefore, it is possible that some of the observed retrogradely labeled neurons resulted from HRP being accidentally deposited in the optic tectum, particularly as the central pretectal nucleus, dorsal accessory optic nucleus, and nucleus isthmi are known to project to the optic tectum in teleosts (Northcutt and Wullimann, '88). However, we believe that the central pretectal and dorsal accessory optic nuclei do indeed project weakly to the valvula cerebelli in Carassius and Lepomis, as we could follow labeled fibers through the anterior mesencephalo-cerebellar tract into the diencephalon.

There appear to be some differences in afferent connections of the valvula in Carassius and Lepomis: A valvulopetal projection from the nucleus of the locus coeruleus was observed in Lepomis but not in Carassius, and valvulopetal projections from the isthmic primary sensory trigeminal nucleus, nucleus prae-eminentialis and the eminentia granularis were seen in Carassius, but not in Lepomis. These differences probably represent real species variations; in particular, the valvulopetal projection from the isthmic primary sensory trigeminal nucleus may be autapomorphic for Carassius. The isthmic primary sensory trigeminal nucleus is the most rostral primary sensory trigeminal area in goldfish (Puzdrowski, '88a), and it is large in Carassius (Fig. 5B) but very small in Lepomis. Application of HRP to the cut ends of the infraorbital and supraorbital nerve trunks containing trigeminal fibers clearly establishes this most rostrally located primary sensory trigeminal nucleus in $L e$ pomis (R.L. Puzdrowski and M.F. Wullimann, unpublished observations). Like carp, goldfish are specialized for sorting food within the oral cavity (Sibbing and Uribe, '85). Although behavioral observations indicate that tactile stimuli are important in this complex process, the exact location of peripheral tactile sensory structures and their innervation have not been established (Jakubowski, '83; Sibbing and Uribe, '85). It is possible that tactile information from the oral cavity reaches the isthmic primary sensory trigeminal nucleus, and the large size of this nucleus and its projection to the valvula cerebelli may reflect an autapomorphy for all similarly specialized cyprinids.

Finger ('78) reported a cerebellopetal "nucleus subeminentialis" located dorsal to the large secondary gustatory nucleus in Ictalurus. In Carassius, the isthmic primary sensory trigeminal nucleus lies rostral to the secondary gustatory nucleus and is labeled by injections into the transitional region between the corpus and valvula cerebelli (Wullimann and Northcutt, '88a) and, especially, by injections into the valvula cerebelli. Finger's ('78) injections of the cerebellum in Ictalurus included all of the corpus and parts of the valvula cerebelli. It is therefore possible that "nucleus subeminentialis" in Ictalurus, like the isthmic primary sensory trigeminal nucleus in Carassius, projects predominantly to the valvula cerebelli. "Nucleus subeminentialis" may therefore be homologous to the isthmic primary sensory trigeminal nucleus.

The existence of sensory trigeminal projections to "nucleus subeminentialis" would support the hypothesis of homology between "nucleus subeminentialis" in Ictalurus and the isthmic primary sensory trigeminal nucleus in Carassius. When HRP was applied to the cut ends of the infraorbital and supraorbital nerve trunks, which carry sensory trigeminal fibers in Ictalurus punctatus (R.L. Puzdrowski and M.F. Wullimann, unpublished observations), transganglionically labeled trigeminal fibers were observed to reach a periventricular isthmic sensory trigeminal nucleus (Fig. 


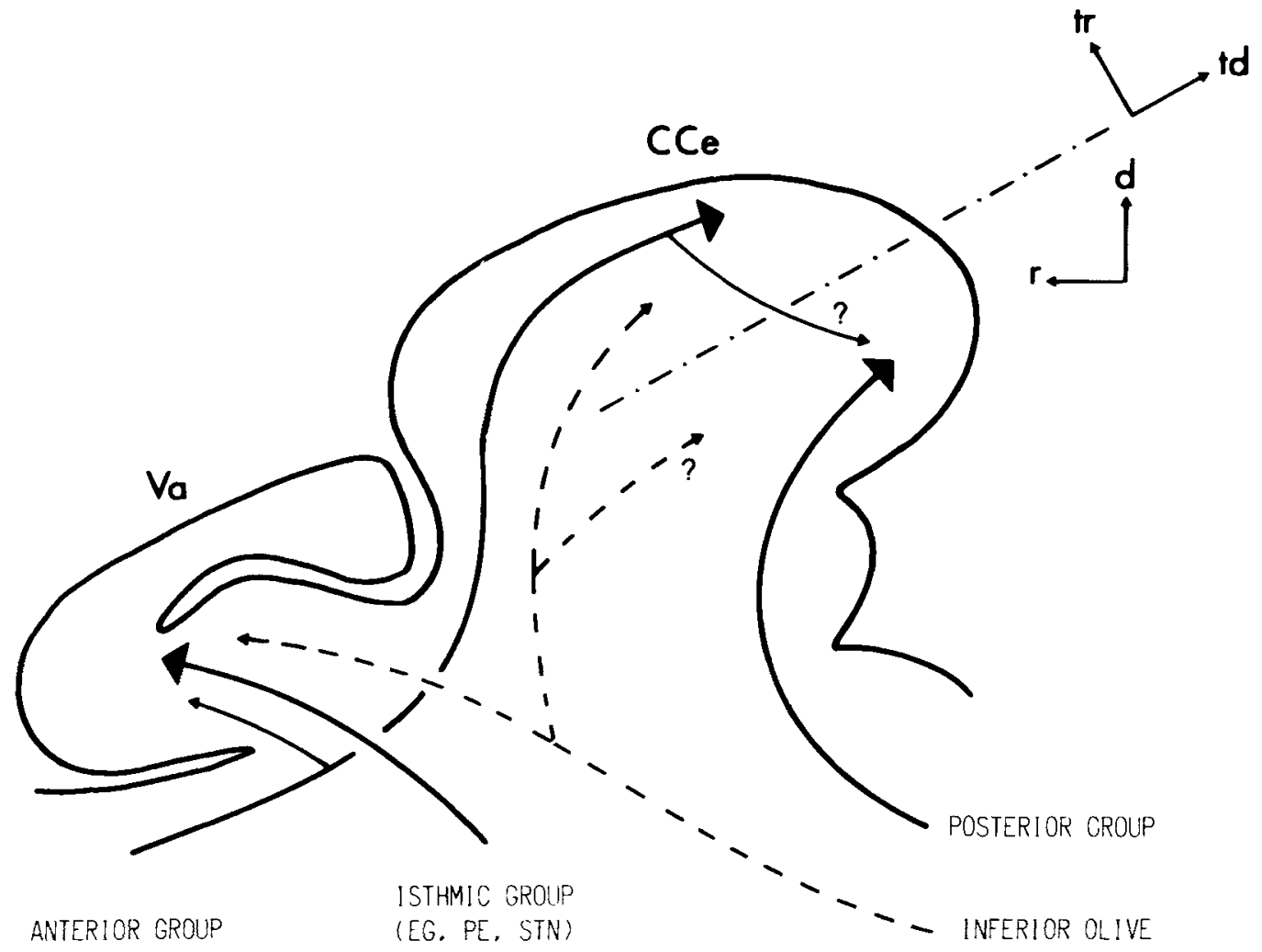

Fig. 8. Differential anatomic inputs to the cerebellum in Carassius. Anterior and posterior groups of corpopetal nuclei, as well as the isthmic group of cerebellopetal nuclei, project to different portions of the cere- bellum. The dorsal and rostral axes are indicated by $\mathbf{d}$ and $\mathbf{r}$, respectively. The distorted axes resulting from the caudal bending of the corpus cerebelli are indicated by td and tr.
9B). More rostrally, some sensory trigeminal fibers form a dorsolaterally directed arch and terminate dorsal to the secondary gustatory nucleus (Fig. 9A), exactly in the position of the "nucleus subeminentialis" described by Finger ('78). It seems clear, therefore, that Finger's "nucleus subeminentialis" is part of the isthmic sensory trigeminal nucleus and represents the homologue of the isthmic primary sensory trigeminal nucleus in Carassius. Its unusual position in Ictalurus is likely due to the relative hypertrophy of the secondary gustatory nucleus in this genus and the consequent dorsomedial displacement of the isthmic sensory trigeminal nucleus.

The nucleus prae-eminentialis is a prominent rhombencephalic structure in all electrosensory teleosts (xenomystines: Braford, '86; mormyrids: Bell and Szabo, '86; gymnotoids: Carr and Maler, '86; siluroids: Finger, '86), and it is intricately related to electroreception. In xenomystines (Braford, '86), siluroids (Tong, '82; Tong and Finger, '83; Finger, '86) and gymnotoids (Carr and Maler, '86), this nucleus comprises two subdivisions. The dorsal part is concerned with electroreception, whereas the ventral part is related to lateral line mechanoreception. Finger ('82) identified nucleus prae-eminentialis for the first time in a nonelectrosensory teleost, the goldfish Carassius auratus. Later, he described the topological position of nucleus praeeminentialis and reported a mechanoreceptive input from the torus semicircularis as well as a projection to the medial octavolateralis nucleus (Finger, '86). However, to our knowledge, nucleus prae-eminentialis has not been described in any other nonelectrosensory teleost. There are two possible evolutionary explanations for its apparent distribution: 1) nucleus prae-eminentialis exists as a rhombencephalic nucleus in all teleosts; it is massively hypertrophied in electrosensory teleosts and inconspicuous in nonelectrosensory teleosts but sufficiently prominent to be recognized in Carassius; 2) nucleus prae-eminentialis arose independently, de novo, in all electrosensory teleosts and some nonelectrosensory teleosts such as Carassius.

Nucleus prae-eminentialis projects to the vestibulolateral lobe of the cerebellum in siluroids, mormyrids, and gymnotoids (Finger, '86: Carr and Maler, '86: Bell and Szabo, '86). In mormyrids, nucleus prae-eminentialis receives a projection from the valvula (Finger et al., ' 81 ) but has not been reported to project to the valvula in this group. It remains to be established whether or not the mechanosensory portion of nucleus prae-eminentialis projects to the valvula cerebelli in electrosensory teleosts, as it does in Carassius. After injections of the valvula in Lepomis, retrogradely labeled cells were not observed in the region where nucleus praeeminentialis lies in Carassius nor, as noted above, could nucleus prae-eminentialis be identified in Lepomis in the normal histological material. Thus, if nucleus prae-eminentialis should turn out to be a primitive feature (plesiomorphy) for teleosts, it is likely that this nucleus has been lost or 


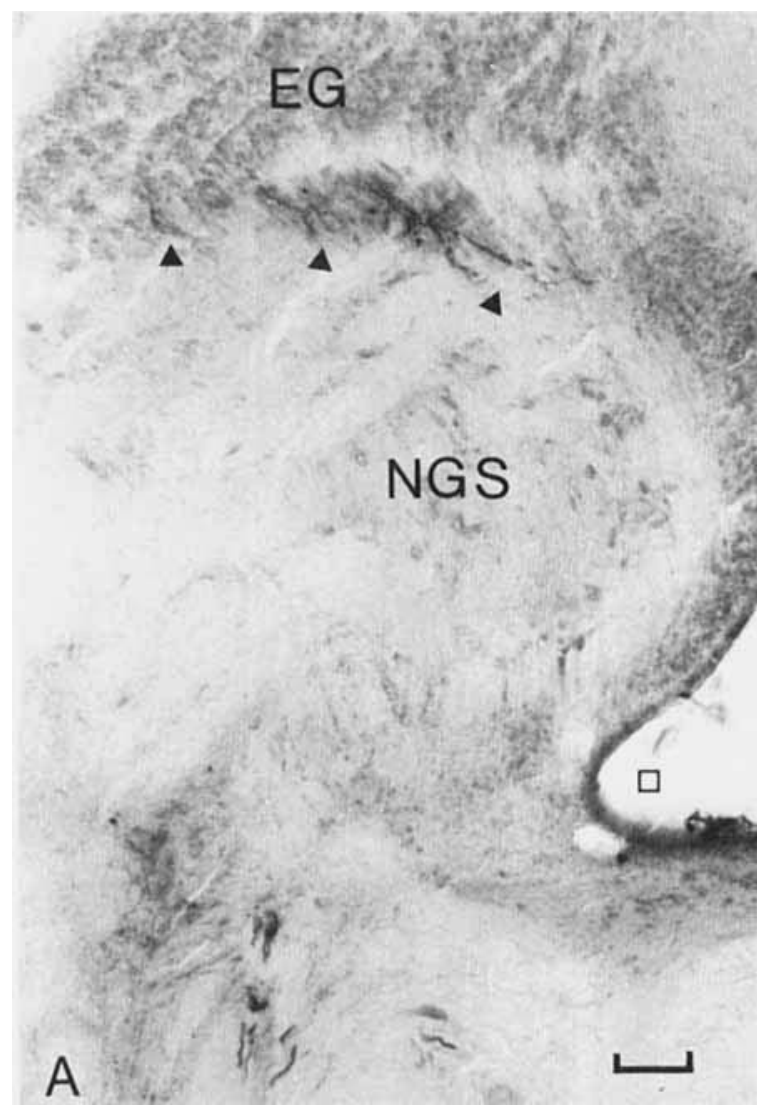

Fig. 9. Cross-sections showing primary trigeminal projections to the isthmic primary sensory trigeminal nucleus in Ictalurus punctatus visualized after HRP application to infraorbital and supraorbital nerve trunks. Although the main sensory projection to the isthmic primary sensory trigeminal nucleus is more medial (B), some sensory trigeminal

reduced in percomorphs. Only future research on higherorder connections of the lateral line system in nonelectrosensory teleosts will clarify the function and phylogenetic distribution of nucleus prae-eminentialis.

The presence of a coerulovalvular projection in Lepomis, and its absence in Carassius, are also supported by immunohistochemical data. Percomorphs, such as Lepomis and Perca (Parent, '83), show a dense catecholaminergic innervation of the granular layer of the valvula cerebelli, whereas the ostariophysine Carassius shows no trace of immunoreactivity in the valvula to antibodies against tyrosine hydroxylase, a catecholamine-synthesizing enzyme (Hornby et al., ' 87 ).

The presence of labeled cells within the corpus cerebelli in Lepomis, and their absence in Carassius, probably do not reflect a real species difference, as we could also follow labeled fibers into the corpus cerebelli within the ipsilateral anterior cerebellar tract after injections of the valvula in Carassius. The labeled cells observed in Lepomis are likely to represent eurydendroid (or basal) cells (Nieuwenhuys and Nicholson, '69b; Nieuwenhuys et al., '74). This unusual intracerebellar connection might indicate that the valvula

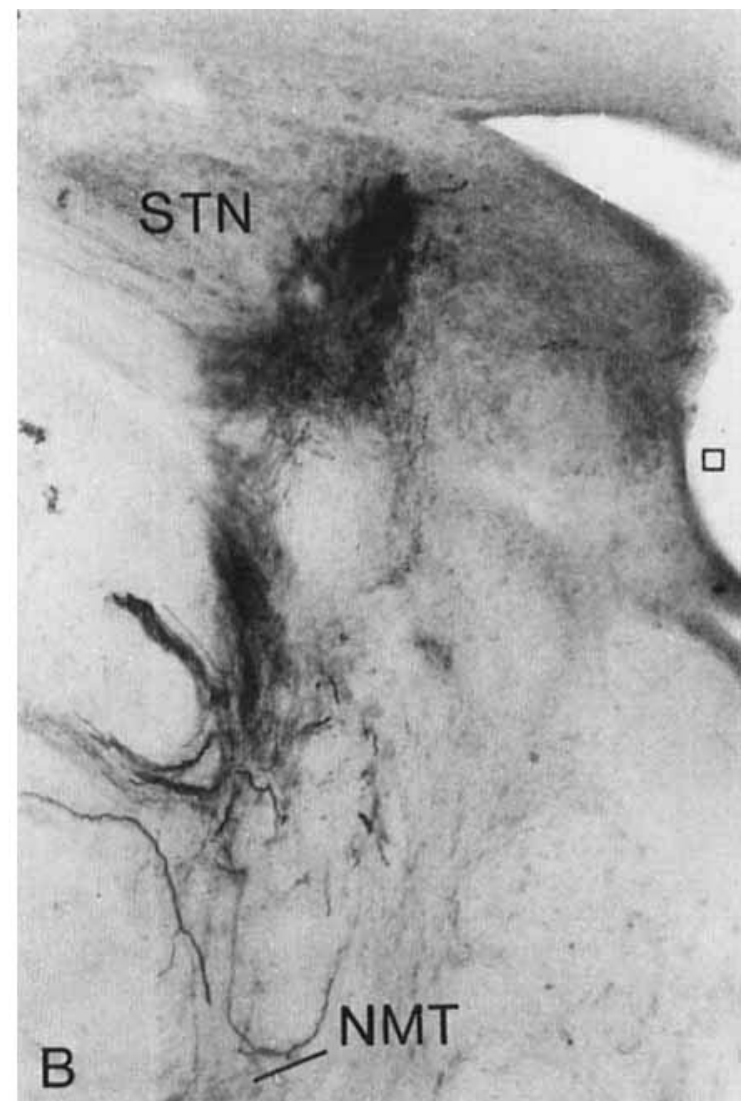

fibers continue to project more rostrodorsally (A, arrowheads) to the level of the secondary gustatory nucleus. Motor fibers turn ventrally toward the motor trigeminal nucleus. In both sections, lateral is to the left and dorsal to the top. The ventricle is indicated with a square. Bar scale $=0.1 \mathrm{~mm}$ for both $\mathrm{A}$ and $\mathrm{B}$.

cerebelli performs at least one higher-order integrative function not performed in the corpus cerebelli.

\section{Are the afferent connections of the valvula cerebelli different in electrosensory and nonelectrosensory teleosts?}

The available anatomic data on afferent connections of the valvula cerebelli in mormyrids (Finger et al., '81; Szabo, '83) indicates that major afferent pathways to the valvula are related to the electric sense. Our data suggest that homologues of these anatomic pathways are absent in Carassius and Lepomis. That is, in these two species, no part of the torus semicircularis projects to the valvula, whereas in Gnathonemus the lateral nucleus of the torus semicircularis (which receives secondary mormyromast and ampullary organ information) and the mediodorsal and medioventral nuclei of the torus semicircularis project to the valvula cerebelli.

Similarly, the dorsal anterior pretectal nucleus of Finger et al., ('81) and the anterior thalamic nucleus of Finger et al. ('81) and Szabo ('83) project to the valvula in mormyrids 
(Finger et al., '81; Szabo, '83). We have argued at length (Wullimann and Northcutt, '87, '88a) that these nuclei are homologues of neural aggregates in the preglomerular area of other teleosts. None of these preglomerular aggregates project to the valvula cerebelli in Carassius or Lepomis, thus indicating that the torovalvular pathway and the preglomerulovalvular pathway are both autapomorphic for mormyrids.

On the other hand, the extensive input to the valvula cerebelli, originating in the isthmic primary sensory trigeminal nucleus in Carassius, has not been reported in Gnathonemus. As noted above, this input may be related to the importance of tactile information during food processing in Carassius. As this connection was not observed in Lepomis either, it might be an autapomorphy for some cyprinids. Only further research on valvular connections in a wide range of teleosts will reveal if the diversity of inputs to the valvula cerebelli seen thus far is the rule or the exception.

\section{Differential anatomical inputs to the cerebellum}

Our results regarding differential anatomic inputs to various parts of the valvula and corpus cerebelli in Carassius (Fig. 8) and Lepomis indicate that different sensory modalities are processed in different parts of the cerebellum. The topologically rostral corpus cerebelli is the subdivision most concerned with vision. It has been shown to receive heavy projections from several primary retinofugal nuclei in the diencephalon and mesencephalon in Lepomis and Carassius: dorsal periventricular pretectal nucleus, central pretectal nucleus, and dorsal and ventral accessory optic nuclei (Northcutt and Wullimann, '88; Wullimann and Northcutt, '88a). This portion of the corpus cerebelli, however, does not appear to receive inputs from those rhombencephalic nuclei that receive primary projections from the lateral line nerve (medial octavolateralis nucleus), trigeminal nerve (nucleus of the descending trigeminal root), and octaval nerve (descending octaval nucleus). These brainstem nuclei project exclusively to the topologically caudal corpus cerebelli (Wullimann and Northeutt, '88a).

Although the valvula cerebelli receives a weak input from two retinorecipient nuclei (central pretectal and dorsal accessory optic nuclei) in Carassius and Lepomis, a major portion of its afferent input in Carassius arises in the isthmic primary sensory trigeminal nucleus and the eminentia granularis, the latter of which receives primary mechanosensory fibers (Puzdrowski, '88b).

Neurophysiological evidence from studies of teleosts supports this pattern of differential sensory inputs to the cerebellum (Kotchabhakdi, '76; Tong and Bullock, '82a; Crispino, '83; Lee and Bullock, '84). In goldfish, visual stimuli elicit responses in the Purkinje cells of the valvula and the anteromedial part of the corpus cerebelli, whereas vestibular stimuli elicit responses in the ventral corpus (Kotchabhakdi, '76). Tactile stimulation of the body surface elicits responses only in the corpus, not in the valvula cerebelli. Because the isthmic sensory trigeminal nucleus receives primary trigeminal projections (Puzdrowski, '88a) and projects heavily to the valvula cerebelli in Carassius, the sensory trigeminal information reaching the isthmic primary sensory trigeminal nucleus must arise from somewhere other than the body surface, perhaps the oral cavity, and the isthmic primary sensory trigeminal nucleus may thus be involved in the complex feeding behavior (Sibbing and Uribe, '85) that some cyprinids exhibit.
In a similar study of the catfish Ictalurus nebulosus, Lee and Bullock ('84) also found that different sensory modalities are processed in different subdivisions of the cerebellum. Although their results differ somewhat from those previously reported in Carassius (Kotchabhakdi, '76), it is clear that the main cerebellar visual area in Ictalurus is also within the corpus cerebelli.

Murakami and Morita ('87) reported that the efferent connections of the teleost cerebellum are also differentially distributed to the various cerebellofugal targets. Most notable is their report that only the valvula cerebelli projects to the lateral nucleus of the valvula. This is in agreement with our finding that the corpus cerebelli does not project to the lateral nucleus of the valvula in Carassius and Lepomis (Wullimann and Northcutt, '88a).

\section{Does the valvula cerebelli have a homologue in cartilaginous fishes?}

In cartilaginous fishes, there are differential anatomic inputs to the anterior lobe and posterior lobe of the corpus cerebelli. In Raja erinacea (Northcutt and Brunken, '84) and Platyrhinoidis triseriata (Fiebig, '88), the retinorecipient cerebellopetal nuclei in the diencephalon (dorsal periventricular pretectal, central pretectal, dorsal and ventral accessory optic nuclei) project only to the anterior lobe and the most rostral part of the posterior lobe, not to the major caudal portion of the posterior lobe, of the corpus cerebelli. Furthermore, electrophysiological evidence (Fiebig, '88; Tong and Bullock, ' $82 \mathrm{~b}$ ) has clearly identified the anterior lobe of the corpus cerebelli as the major visual center and the posterior lobe as the tactile, vestibular, and electrosensory center in Platyrhinoidis.

Given this anatomic and neurophysiologic data for elasmobranchs, it seems likely that the topologically rostral corpus cerebelli in teleosts is homologous to the anterior lobe of the corpus cerebelli in cartilaginous fishes. Similarly, the topologically caudal part of the corpus cerebelli in teleosts is most likely homologous to the posterior lobe of the corpus cerebelli in cartilaginous fishes. It would appear that the valvula cerebelli represents an evolutionarily derived structure shared by all ray-finned fishes (synapomorphy) and that it has no homologue in cartilaginous fishes, based on the following considerations:

1. The topologically rostral part of the corpus cerebelli in teleosts, like the anterior lobe of the corpus cerebelli in elasmobranchs, is the main visual cerebellar center, not the valvula cerebelli.

2. In Carassius, the major inputs to the valvula cerebelli originate in structures that are autapomorphic for teleosts (eminentia granularis, nucleus prae-eminentialis) and are not present in cartilaginous fishes.

3. The valvula cerebelli receives different anatomic inputs in various teleosts, which may be related to the relative importance of different sensory systems in different species.

In summary, the valvula cerebelli in teleosts differs from the anterior lobe of the cerebellar corpus in cartilaginous fishes not only in its topological position ventral to the optic tectum, but also in its afferent connections and neurophysiology. Because of these differences, it is unlikely that the valvula cerebelli represents the homologue of the anterior cerebellar lobe. Furthermore, the topologically rostral part of the corpus cerebelli in teleosts shares numerous similarities with the anterior cerebellar lobe in cartilaginous fishes, and we thus recognize it as the homologue. 


\section{ACKNOWLEDGMENTS}

This work was supported by a Swiss National Science Foundation fellowship to MFW and NIH grants NS24669 and NS24869 to RGN. The authors thank Mary Sue Northcutt, Richard L. Pudzdrowski, George F. Striedter, and Jacqueline $\mathrm{F}$. Webb for reading and commenting on various versions of the manuscript.

\section{LITERATURE CITED}

Bell, C.C., and T. Szabo (1986) Electroreception in mormyrid fish. Central anatomy. In T.H. Bullock and W. Heiligenberg (eds): Electroreception. New York: Wiley, pp. 375-421.

Braford, M.R. Jr. (1986) African knivefishes. The xenomystines. In T.H. Bullock and W. Heiligenberg (eds): Electroreception. New York: Wiley, pp. 453-464.

Braford, M.R. Jr., and R.G. Northcutt (1983) Organization of the diencephalon and pretectum of the ray-finned fishes. In R.E. Davis and R.G. Northcutt (eds): Fish Neurobiology, Vol. 2. Ann Arbor: University of Michigan Press, pp. 117-163.

Bullock, T.H., D.A. Bodznick, and R.G. Northcutt (1983) The phylogenetic distribution of electroreception: Evidence for convergent evolution of a primitive vertebrate sense modality. Brain Res. Rev. 6:25-46.

Carr, C.E., and L. Maler (1986) Electroreception in gymnotiform fish. Central anatomy and physiology. In T.H. Bullock and W. Heiligenberg (eds): Electroreception. New York: Wiley, pp. 319-373.

Crispino, L. (1983) Modification of responses from specific sensory systems in midbrain by cerebellar stimulation: Experiments on a teleost fish. J. Neurophysiol. 49:3-15.

Fiebig, E. (1988) Connections of the corpus cerebelli in the thornback guitarfish, Platyrhinodis triseriata (Elasmobranchii). J. Comp. Neurol. 268:567-583.

Finger, T.E. (1978) Cerebellar afferents in teleost catfish (Ictaluridae). J. Comp. Neurol. 181:173-182.

Finger, T.E. (1982) Connections of the lateral line lobe (n. medialis) in goldfish, Carassius auratus. Soc. Neurosci. Abstr. 8:763.

Finger, T.E. (1983) Organization of the teleost cerebellum. In R.G. Northcutt and R.E. Davis (eds): Fish Neurobiology, Vol. 1. Ann Arbor: University of Michigan Press, pp. 261-284.

Finger, T.E. (1986) Electroreception in catfish. Behavior, anatomy, and Electrophysiology. In T.H. Bullock and W. Heiligenberg (eds): Electroreception. New York: Wiley, pp. 287-317.

Finger, T.E., C.C. Bell, and C.J. Russell (1981) Electrosensory pathways to the valvula cerebelli in mormyrid fish. Exp. Brain Res. 42:23-33.

Gould, S.J., and E.S. Vrba (1982) Exaptation-a missing term in the science of form. Palaeobiology 8:4-15.

Hanker, J.S., P.E. Yates, C.B. Metz, and A. Rustioni (1977) A new specific, sensitive and non-carcinogenic reagent for the demonstration of horseradish-peroxidase. Histochem. J. 9:789-792.

Hornby, P.J., D.T. Piekut, and L.S. Demski (1987) Localization of immunoreactive tyrosine hydroxylase in the goldfish brain. J. Comp. Neurol. $261: 1-14$.

Jakubowski, M. (1983) New details of the ultrastructure (TEM, SEM) of taste buds in fishes. Z. Mikrosk.-Anat. Forsch. 97:849-862.

Kotchabhakdi, N. (1976) Functional organization of the goldfish cerebellum. J. Comp. Physiol. 112:75-93.

Larsell, O. (1967) The Comparative Anatomy and Histology of the Cerebellum from Myxinoids Through Birds. Minneapolis: University of Minnesota Press, pp. 291.

Lauder, G.V., and K.F. Liem (1983) The evolution and interrelationships of the actinopterygian fishes. Bull. Mus. Comp. Zool. 150:95-197.

Lee, L.T., and T.H. Bullock (1984) Sensory representation in the cerebellum of the catfish. Neuroscience 13:157-169.

Mesulam, M.M. (1978) Tetramethyl benzidine for horseradish peroxidase neurohistochemistry. A non-carcinogenic blue reaction-product with su- perior sensitivity for visualizing neural afferents and efferents. J. Histochem. Cytochem. 26:106-117.

Murakami, T., and Y. Morita (1987) Morphology and distribution of the projection neurons in the cerebellum in a teleost, Sebastiscus marmoratus. J. Comp. Neurol. 256:607-623.

Nieuwenhuys, R. (1967) Comparative anatomy of the cerebellum. Prog. Brain Res. 25:1-93.

Nieuwenhuys, R. (1982) An overview of the organization of the brain of actinopterygian fishes. Am. Zool. 22:287-310.

Nieuwenhuys, R., and C. Nicholson (1969a) A survey of the general morphology, the fiber connections, and the possible functional significance of the gigantocerebellum of mormyrid fishes. In R. Llinas (ed): Neurobiology of Cerebellar Evolution and Development. Chicago: American Medical Association, pp. 107-134.

Nieuwenhuys, R., and C. Nicholson (1969b) Aspects of the histology of the cerebellum of mormyrid fishes. In R. Llinas (ed): Neurobiology of cerebellar evolution and development. Chicago: American Medical Association, pp. 135-169.

Nieuwenhuys, R., E. Pouwels, and E. Smulders-Kersten (1974) The neuronal organization of cerebellar lobe $\mathrm{C} 1$ in the mormyrid fish Gnathonemus petersii (Teleostei). Z. Anat. Entwickl.-Gesch. 144:315-336.

Northcutt, R.G. (1977) Elasmobranch central nervous system organization and its possible evolutionary significance. Am. Zool. 17:411-429.

Northcutt, R.G. (1978) Brain organization in the cartilaginous fishes. In E.S. Hodgson and R.F. Mathewson (eds): Sensory Biology of Sharks, Skates and Rays. Arlington: Office of Naval Research, pp. 117-193.

Northcutt, R.G., and W.J. Brunken (1984) Cerebellar afferents in the little skate (Batoidea). Soc. Neurosci. Abstr. 10:859.

Northcutt, R.G., and M.F. Wullimann (1988) The visual system in teleost fishes: Morphological patterns and trends. In J. Atema, R.R. Fay, A.N. Popper, and W.N. Tavolga (eds): Sensory Biology of Aquatic Animals. New York: Springer, pp. 515-552.

Parent, A. (1983) The monoamine-containing neurongl systems in the teleostean brain. In R.E. Davis and R.G. Northcutt (eds): Fish Neurobiology, Vol. 2. Ann Arbor: University of Michigan Press, pp. 285-315.

Puzdrowski, R.L. (1988a) Afferent projections of the trigeminal nerve in goldfish, Carassius auratus. J. Morphol. 198:131-148.

Puzdrowski, R.L. (1988b) The peripheral distribution and central projections of the trigeminal, facial and lateral line nerves in goldfish, Carassius auratus. Ph.D. Dissertation, University of Michigan, Ann Arbor.

Russell, C.J., and C.C. Bell (1978) Neuronal responses to electrosensory input in mormyrid valvula cerebelli. J. Neurophysiol. 41:1495-1510.

Sibbing, F.A., and R. Uribe (1985) Regional specializations in the oro-pharyngeal wall and food processing in the carp (Cyprinus carpio L.) Neth.J. Zool. 35:377-422.

Szabo, T. (1983) Cerebellar pathways in the brain of the mormyrid teleost fish. Acta Morphol. Acad. Sci. Hung. 31:219-234.

Tong, S.-L. (1982) The nucleus praeeminentialis: An electro- and mechanoreceptive center in the brainstem of the catfish. J. Comp. Physiol. 145:299309.

Tong, S.-L., and T.H. Bullock (1982a) Electroreceptive representation and its dynamics in the cerebellum of the catfish, Ictalurus nebulosus (Ictaluridae, Siluriformes). J. Comp. Physiol. 145:289-298.

Tong, S.-L., and T.H. Bullock (1982b) The sensory functions of the cerebellum of the thornback ray, Platyrhinoidis triseriata. J. Comp. Physiol. 148:399-410.

Tong, S.-L., and T.E. Finger (1983) Central organization of the electrosensory lateral line system in bullhead catfish Ictalurus nebulosus. J. Comp. Neurol. 217:1-16.

Wullimann, M.F., and R.G. Northcutt (1987) Telencephalic and retinal pro jections allow reinterpretation of the diencephalon in mormyrids. Soc. Neurosci. Abstr. 13:130.

Wullimann, M.F., and R.G. Northcutt (1988a) Connections of the corpus cerebelli in the green sunfish and the common goldfish: A comparison of perciform and cypriniform teleosts. Brain Behav. Evol. 32:293-316.

Wullimann, M.F., and R.G. Northcutt (1988b) Afferent connections of the valvula cerebelli in the goldfish, Carassius auratus. Fortschr. Zool. (Abstract) (in press). 